BRX TH-565

\title{
Birkhoff for Lovelock Redux
}

\author{
S. Deser ${ }^{1}$ and J. Franklin ${ }^{2}$ \\ ${ }^{1}$ Department of Physics, Brandeis University \\ Waltham, MA 02454, USA \\ deser@brandeis.edu \\ ${ }^{2}$ MIT Kavli Institute for Astrophysics and Space Research \\ Cambridge, MA 02139, USA \\ jfrankli@mit.edu
}

\begin{abstract}
We show succinctly that all metric theories with second order field equations obey Birkhoff's
\end{abstract} theorem: their spherically symmetric solutions are static.

The special, "Lovelock", metric theories with second derivative order field equations (relevant 
details, and the original references, may be found in [1], whose notation we use.) obey Birkhoff's theorem: their spherically symmetric solutions are static $[2,3]$. In this note, we provide an alternative derivation of the theorem, using the approach of [4], where historical references-and justification of our shortcuts-are given. We also show that the apparent counterexamples to Birkhoff in tuned combinations of Lovelock actions found in $[2,3]$ are merely deSitter vacua, such as can always be introduced by tuning any action polynomial in curvatures.

We will focus on the single relevant, (0r), "radial energy flux" field equation, using the metric ansatz

$$
d s^{2}=-a b^{2} d t^{2}+a^{-1} d r^{2}+r^{2} d_{n} \Omega+2 f b d r d t
$$

where $(a, b, f)$ depend on $(r, t)$ and $d_{n} \Omega$ is the unit $n$-sphere interval. As we shall see, the seemingly superfluous $f \sim g_{0 r}$ component's variation provides precisely the required field equation. The Lovelock actions of order $k$ in $D=n+2$ dimensions are defined as

$$
I_{k}=\int d^{D} x \mathcal{L}_{k} \equiv \int d^{D} x(-g)^{-1 / 2} \epsilon^{\mu_{1} \ldots} \epsilon^{\nu_{1} \ldots} R_{\mu_{1} \mu_{2} . .} \ldots R_{\nu_{k-1} \nu_{k} .} g_{. .} \ldots g .
$$

There are $k$ curvatures, whose $4 k$ indices contract with the $2 \mathrm{D} \epsilon$-indices; the indicated metrics soak up the rest. $\mathcal{L}_{k}$ vanishes for $D<2 k$ and $I_{k}$ is a topological invariant at $D=2 k$. The 
Palatini, $\delta R \sim D D \delta g$, and Bianchi, $R_{\mu[\nu \alpha \beta ; \lambda]} \equiv 0$, identities guarantee that only the variations of the explicit metrics (but not those of the $R$ 's) survive, leading to field equations $\mathcal{G}_{\mu \nu}=0$ algebraic of order $k$ in the curvatures but without explicit derivatives. The leading, $k=0,1,2$ actions are the cosmological, GR and Gauss-Bonnet terms. The form of every $I_{k}[a, b]$, upon inserting the above metric, first at $f=0$, is $[1]$

$$
I_{k}[a, b] \sim \int d r b\left[r^{n+1} \psi^{k}\right]^{\prime}, \quad r^{2} \psi=(1-a)
$$

where primes denote radial- (and dots will mean time-) derivatives. Note first that, even though we have not assumed time independence of $(a, b)$, only spatial derivatives (that there is only one prime, rather than two, is peculiar to Schwarzschild gauge.) survive [5]. Further, (3) will be unaffected by subsequent inclusion of $f$, since the latter will only be used as a Lagrange multiplier to deliver the $(0 r)$ equation, then set to zero, to recover the 2 -function $(f=0)$ metric. We learn from varying $(3)$ with respect to $a$ that $b=b(t)$, which means it can be removed entirely by using the residual timegauge choice $b(t) d t=d \tilde{t}$. [Thence our unusual, but legitimate, parametrization of $d s^{2}$.] Varying with respect to $b$ fixes $a(r, t)$, e.g. $a=1-2 m(t) / r^{n-1}$ in GR; the notation emphasizes that $m$ 's 
time-dependence is as yet undetermined. For that, we need the final, $f$-field equation,

$$
\left.\frac{\delta I_{k}[a, f]}{\delta f}\right|_{f=0} \equiv F_{k-1}(a) \dot{a}=0
$$

where we can use the now known $(a, b)$ to remove $b$ altogether and as explained, $f$ has also been set to 0 after variation. We have-legitimately-anticipated the form of this equation, simply because it must have exactly one time derivative and only depends on $a(r, t)$. So Birkhoff is proved, unless $F_{k-1}(a)=0$, which is certainly impossible for any one $k$ or we would have lost an entire field equation! We will show below that this is also the case for all linear combinations, $\sum_{k} c_{k} I_{k}$. So the only work required is to justify that (4) follows from (2) at $b=1, f=0$. In varying the action to get its $(0 r)$ part, we need not keep the explicit $(-g)^{-1 / 2}$, since $\delta \ln (-g)=g^{\mu \nu} \delta g_{\mu \nu}$. The $(D-2 k) g_{\mu \nu}$ factors of $(2)$ all vary identically, so we find

$$
\left.\frac{\delta I_{k}}{\delta g_{0 r}} \equiv \mathcal{G}^{0 r} \sim \epsilon^{0 \cdots} \epsilon^{r \cdots g} \ldots g R \ldots R\right|_{f=0}=0
$$

Because $g_{0 i}=0$, the remaining $g_{\alpha \beta}$ must all be spatial (since only one " 0 " index is available); then all curvatures but one are purely spatial, the remaining one being $\sim R_{0 i j k}$.

For orientation, let us briefly consider (5) in Riemann normal coordinates: the $R_{i j k \ell}$ are proportional to $a$ and its radial derivative, while $R_{0 i j k} \sim\left(\dot{g}_{i j, k}-\dot{g}_{i k, j}\right)$ has a single $\partial / \partial t$. Hence, we 
are morally assured that the "guess" (4) is correct, and indeed that $F_{k-1}(a)$ is just the $(k-1)^{\text {th }}$ power of $F_{1}=(1-a)$, the Einstein value. The Appendix confirms this: all spatial curvature components depend on $(1-a)$ and $(1-a)^{\prime}$.

Finally, we turn to generic Lovelock combinations whose actions are of the form [1]

$$
I=\sum_{k=0}^{N} c_{k} I_{k}=\sum_{k=0}^{N} c_{k} \int d r b\left(r^{n+1} \psi^{k}\right)^{\prime}, \quad r^{2} \psi=(1-a)
$$

plus terms linear in $f$. Clearly, $b=b(t)$ again and gets absorbed by a change of $t$ while $\psi$ obeys

the algebraic equation $\sum c_{k} \psi^{k}=m(t) r^{-(n+1)}$. Finally, as predicted, the "Birkhoff equation", is

$$
\left(\sum_{k=1}^{N} d_{k} \psi^{k-1}\right) \dot{\psi}=0
$$

the $d_{k}$ differ from the original constants $c_{k}$ by constant numerical factors. As we noted, a cosmological term $(k=0)$ would not contribute explicitly. So $\psi$ is time-constant unless the prefactor in (7) vanishes. This vanishing was interpreted in [2,3], in a different gauge, to imply that there are Birkhoff-violating solutions. We now see instead that the theorem always holds, and that these exceptional geometries are nothing but (anti) deSitter vacua that generalize the original flat one by fine-tuning the $c_{k}$ : From (7), the zeros of the polynomial are its (necessarily time-constant) roots, $\psi_{i}=R_{i}$, namely the constant curvature metrics $a_{i}=1+R_{i} r^{2}$. These deSitter spaces not 
only obey Birkhoff, but the effective cosmological constants can be written, upon inserting the $a_{i}$ into the $a$-field equation, in terms of the $c_{k}$ coefficients, including (if any) the original $c_{0}=\Lambda$.

Their significance as additional vacua is understood as follows: Suitable choices of the constants

in any $I=\sum \gamma_{k} R^{k}$, Lovelock or not, can be fine-tuned to allow constant curvature spaces for any

desired $\Lambda$. These vacua actually agree with the seemingly time-dependent spaces of $[2,3]$ upon

transforming the latter to Schwarzschild gauge, namely choosing the coefficient of $d_{n} \Omega$ to be $r^{2}$.

Finally, this also shows that we have not lost a field equation here either-it has just "solved itself"

to yield deSitter!

\section{Appendix}

In this Appendix, we first provide the form of the Riemann tensor for our time-dependent metric

ansatz; it depends on five functions, reducing to [1] for $\dot{a}=\dot{b}=0$ :

$$
\begin{aligned}
R_{\mu \nu \alpha \beta} & =4 A \delta_{[\mu}^{0} \delta_{\nu]}^{r} \delta_{[\alpha}^{0} \delta_{\beta]}^{r}+4 B Z_{i} \delta_{[\mu}^{0} \delta_{\nu]}^{\theta_{i}} \delta_{[\alpha}^{0} \delta_{\beta]}^{\theta_{i}}+4 C Z_{i} \delta_{[\mu}^{r} \delta_{\nu]}^{\theta_{i}} \delta_{[\alpha}^{r} \delta_{\beta]}^{\theta_{i}}+2 r^{4} \psi Z_{i} Z_{j} \delta_{[\mu}^{\theta_{i}} \delta_{\nu]}^{\theta_{j}} \delta_{[\alpha}^{\theta_{i}} \delta_{\beta]}^{\theta_{j}} \\
& +4 D Z_{i}\left(\delta_{[\mu}^{0} \delta_{\nu]}^{\theta_{i}} \delta_{[\alpha}^{r} \delta_{\beta]}^{\theta_{i}}+\delta_{[\mu}^{r} \delta_{\nu]}^{\theta_{i}} \delta_{[\alpha}^{0} \delta_{\beta]}^{\theta_{i}}\right)
\end{aligned}
$$


with

$$
\begin{aligned}
& A=\frac{b}{2}\left[3 a^{\prime} b^{\prime}+a^{\prime \prime} b+2 a b^{\prime \prime}\right]+\frac{1}{2 a^{2}}\left[-\frac{\dot{a} \dot{b}}{b}+\frac{a \ddot{a}-2(\dot{a})^{2}}{a}\right] \\
& B=\frac{1}{2}(r a b)\left[a^{\prime} b+2 a b^{\prime}\right] \\
& C=-\frac{a^{\prime} r}{2 a}, \quad D=-\frac{\dot{a} r}{2 a}, \quad \psi=\frac{1-a}{r^{2}}
\end{aligned}
$$

and $Z_{i}$ is the $i^{\text {th }}$ angular component of the metric.

We are interested in relating $\mathcal{G}^{0 r}(k)$ and $\mathcal{G}^{0 r}(k-1)$ to exhibit their "polynomial in $\psi$ " nature, outlining the steps here. We find that effectively, the Riemann tensor appears in the field equation in the combination

$$
\tilde{R}_{\alpha \beta \gamma \rho}=2 \psi g_{\alpha \beta} g_{\gamma \rho}+4 D r^{-2} g_{\gamma \rho} \delta_{\alpha}^{r} \delta_{\beta}^{0}
$$

The first term above is $\mathcal{G}^{0 r}(k-1)$ while the second is (in odd dimensions) $k^{-1} G^{0 r}(k)$ when replacing the $k^{\text {th }}$ Riemann tensor in the field equation (5). After some algebra, this leads to

$$
\mathcal{G}^{0 r}(k)=2 \psi \mathcal{G}^{0 r}(k-1)+k^{-1} \mathcal{G}^{0 r}(k) \rightarrow \mathcal{G}^{0 r}(k)=\frac{2 k \psi}{k-1} \mathcal{G}^{0 r}(k-1),
$$

and so

$$
\mathcal{G}^{0 r}(k)=(\text { const. }) \times \psi^{k-1} \mathcal{G}^{0 r}(1)
$$


the final term here is Einstein, and contains a factor of $D$ and a product of all the angular depen-

dence but no $\psi$.

Our other task here is, for concreteness, to show explicitly in $\mathrm{D}=5$ how the seeming obstructions to Birkhoff are really deSitter vacua, as shown generically in text.

For the metric ansatz:

$$
d s^{2}=-a b^{2} d t^{2}+a^{-1} d r^{2}+2 b f d t d r+r^{2}\left(d \psi^{2}+\sin ^{2} \psi\left(d \theta^{2}+\sin ^{2} \theta d \phi^{2}\right)\right)
$$

we form the cosmological, Einstein, and Gauss-Bonnett actions

$$
I=\int d^{5} x \sqrt{-g}\left(\Lambda+R+\alpha\left(R^{2}-4 R^{\mu \nu} R_{\mu \nu}+R^{\mu \nu \alpha \beta} R_{\mu \nu \alpha \beta}\right)\right),
$$

keeping only terms linear in $f$ and its derivatives. The field equations at $f=0$ read:

$$
\begin{aligned}
\left.\frac{\delta I}{\delta a}\right|_{f=0} & =3\left(4 \alpha(1-a)+r^{2}\right) b^{\prime}=0 \\
\left.\frac{\delta I}{\delta b}\right|_{f=0} & =\Lambda r^{3}+6 r(1-a)-3 r^{2} a^{\prime}-12 \alpha a^{\prime}(1-a) \\
\mathcal{G}^{0 r} & \left.\equiv \frac{\delta I}{\delta f}\right|_{f=0}=-3\left(r^{2}+4 \alpha(1-a)\right) \dot{a} a^{-1} .
\end{aligned}
$$

Now consider the obstruction, $4 \alpha(1-a)+r^{2}=0$, to Birkhoff. This is just $a=1+\frac{r^{2}}{4 \alpha}$, vacuum 
deSitter. The constant $4 \alpha$ is determined by inserting into the $b$ equation:

$$
\left.\frac{\delta I}{\delta b}\right|_{f=0, a=1+r^{2} /(4 \alpha)}=\frac{r^{3}(2 \alpha \Lambda-3)}{2 \alpha}=0
$$

The Lovelock coefficients determine the deSitter cosmological constant according to the tuning $\alpha=3 \Lambda / 2$; in the absence of $\Lambda$, there are no roots, except flat space.

We thank B. Tekin for a discussion. This work was supported by NSF grant PHY-04-01667.

\section{References}

[1] S. Deser and A.V. Ryzhov; gr-qc/0505039.

[2] C. Chermousis and J.F. Dufaux, Class. Quant. Grav. 19, 4671 (2002); hep-th/0202107.

[3] R. Zegers; gr-qc/0505016.

[4] S. Deser and J. Franklin, Am. J. Phys. 73, 261 (2005); gr-qc/0408067.

[5] S. Deser and B. Tekin, Class. Quant. Grav. 20. 4877 (2003); gr-qc/0306114. 